\title{
Some No Longer Unknowns in Science
}

\author{
Arieh Ben-Naim \\ Department of Physical Chemistry, The Hebrew University of Jerusalem, Jerusalem, Israel \\ Email: arieh@fh.huji.ac.il
}

Received October 26, 2011; revised November 26, 2011; accepted December 5, 2011

\begin{abstract}
Three out of 125 "big questions of science", can now be claimed to have been answered. All of these questions involve water; its structure, its role in protein folding and its role in protein-protein association.
\end{abstract}

Keywords: Structure of Water; Protein Folding; Protein-Protein Association; Solubility of Proteins

In the July 2005 issue of Science [1], the editors Kennedy and Norman listed 125 big questions that "point out to critical knowledge gaps." It is the purpose of this communication to bring to the attention of the readers of this Journal that three out of the 125 questions are already very well answered, and understood. The three questions which we are addressing here are [1]:

a) What is the structure of water? Researchers continue to tussle over how many bonds each $\mathrm{H}_{2} \mathrm{O}$ molecule makes with its nearest neighbors.

b) Can we predict how proteins will fold? Out of a near infinitude of possible ways to fold, protein picks one in just tens of microseconds. The same task takes 30 years of computer time.

c) How do proteins find their partners? Protein-protein interactions are at the heart of life. To understand how partners come together in precise orientations in seconds, researchers need to know more about the cell's biochemistry and structural organization.

These three questions are intimately related to each other. Although that is not stated explicitly in the questions, it will become clear that the properties of water harbor the clues to the answers of all three questions [2,3]. The first part of question (a) is concerned with the "structure of water" which has been defined in several ways [2]. The second part refers to the number of bonds each water molecules makes. The answer to the latter question is simple; at each temperature and pressure there are water molecules engaged in zero, one, two, three or four bonds. The average number of bonds in which a single molecule is engaged depends on the temperature and pressure of the water. This is a statistically average quantity, and is related to the "structure of water" only if we define the structure of water as the average number of bonds formed by a single water molecule at any given temperature and pressure.
In my opinion the main mystery associated with liquid water is not the "structure of water," nor the average number of bonds, but the outstanding properties of liquid water. These properties are by large well known and were well understood even before 2005 [2]. Therefore, the inclusion of this question in the list of "unknowns of Science" was unwarranted even in 2005.

Regarding the second question (b), the best formulation of the problem, as well as a hint for a solution to the problem were provided by Levinthal in 1968 [4,5]. This problem became a formidable challenge for many scientists. Unfortunately, almost all efforts in solving this problem were misdirected, and therefore reached a deadend.

The main culprits for the misdirected efforts are a result of the misinterpretation of the so-called Anfinsen's Thermodynamic Hypothesis [6]. The Anfinsen's hypothesis is often referred to as Anfinsen's Dogma [7], and includes two parts.

a) The native structure of a protein is determined by the sequence of the amino acids.

b) The native structure of a protein is at a minimum Gibbs energy.

The two parts of Anfinsen's Dogma, (i) and (ii) are correct. Unfortunately, many scientists conjectured that (i) implies the existence of a code, that translates from the sequence of amino acids to the 3-D structures. Some inferred from the second part (ii) that the Gibbs energy landscape must have a global minimum, an inference which is unwarranted by the Second Law of Thermodynamics $[3,7]$. Yet, some others used ideas from Evolution theory to explain the process of protein folding [8].

All these approaches may be referred to as targetbased theories. The inadequacies of these approaches were discussed elsewhere [3]. Even those that sought the more plausible caused-based solutions to the problem of pro- 
tein folding, went astray by adhering to the hydrophobic dominance dogma [3]. This hypothesis, originally suggested by Kauzmann in 1959, states that the major factor that "speeded and guided" [4] the folding of protein is the hydrophobic effect [9]. Note however, that Kauzmann himself was cautious in claiming only that the "hydrophobic bond is probably one of the most important factors..."

Again, it is unfortunate that so much effort had been expanded in studying the hydrophobic effect which did not, and could not deliver any strong forces acting on the groups of the protein [3].

All these have changed when strong hydrophilic forces were discovered in 1990 [3,10,11], which provided a sound cause-based approach to protein folding, as well as an answer to Levinthal's question.

Briefly, the answer to the question of how protein folds to a precise 3D structure in a very short time is straightforward. At each stage of the folding process strong hydrophilic forces act on the hydrophilic groups of the protein and force the protein to fold in a narrow range of preferential pathways. The stronger the forces, the narrower the range of pathways, and the shorter the time to reach the final 3D structure. Thus, the seeds of the solution to the protein folding problem were already included in Levinthal's answer $[4,5]$. The discovery of the hydrophilic forces provides a compelling cause-based answer of the Levinthal's question [3], hence, also an answer to the question (b).

The third question (c) includes two parts: one is to find the factors that stabilize the protein aggregates, and the second involves the factors that determine the specificity of the binding mode. This is also known as the problem of molecular recognition [3,12]. As in the case of protein folding, the adherence to the hydrophobic dominance has led scientists to seek solutions to the problem of selfassembly in the wrong direction. This situation has been radically changed once the hydrophilic interactions were discovered. These interactions provided answers to both the stability of aggregates of proteins, as well as to the specificity of the mode of binding $[3,12,13]$. Therefore, question (c) has also been answered.

Finally, I would like to mention another question which although not listed among the 125 "Big questions" is not less of a mystery than either of the two questions (b) and (c) involving protein. The question is "why are globular proteins very soluble in aqueous solutions" [3]. Again, the answer to this until-recently "unknown of science" involves the strong hydrophilic interactions. Everyone who has given a thought regarding the question of the reasons for the large solubility of globular proteins, correctly concluded that the hydrophilic groups on the surface of the proteins are responsible for its solubility. What was less known is that not the mere number of hydrophilic groups that are exposed to the solvent determine its high solubility, but the specific distribution of the hydrophilic groups on the surface of the protein [3]. It was recently shown that the possibility of formation of water-bridges between two or more hydrophilic groups contributes significantly, if not decisively to the large solubility of the protein $[3,14]$. Therefore, I believe that the problem of the large solubility of the globular proteins should have been included in the list at the time the questions (b) and (c) were included [1], only to be deleted later on along with problems (b) and (c).

\section{Acknowledgements}

The author is grateful for comments and suggestions from Robert Baldwin, David Eisenberg, Robert Mazo and Mihaly Mezei.

\section{REFERENCES}

[1] D. Kennedy and C. Norman, "What Don’t We Know?" Science, Vol. 309, No. 5731, 2005, p. 75. doi:10.1126/science.309.5731.75

[2] A. Ben-Naim, "Molecular Theory of Water and Aqueous Solutions, Part I: Understanding Water,” World Scientific, Singapore, 2009. doi:10.1142/9789812837615

[3] A. Ben-Naim, "Molecular Theory of Water and Aqueous Solutions: Part II The Role of Water in Protein Folding Self Assembly and Molecular Recognition,” World Scientific, Singapore, 2011.

[4] C. Levinthal, "Are There Pathways for Protein Folding," Journal de Chimie Physique, Vol. 65, No. 1, 1968, pp. 44-45.

[5] C. Levinthal, "Mossbauer Spectroscopy in Biological Systems,” J. T. P. De Brunner and E. Munck, Eds., University of Illinois Press, 1969, pp. 22-24.

[6] C. B. Anfinsen, "Principles that Govern the Folding of Protein Chains," Science, Vol. 181, No. 4096, 1973, pp. 223-230. doi:10.1126/science.181.4096.223

[7] A. Ben-Naim, "Pitfalls in Anfinsen's Thermodynamic Hypothesis," Chemical Physics Letters, Vol. 511, No. 1-3, 2011, pp. 126-128. doi:10.1016/j.cplett.2011.05.049

[8] R. Zwanzig, A. Szabo and B. Bagchi, "Levinthal’s Paradox," Proceedings of the National Academy of Science, Vol. 89, No. 1, 1992, pp. 20-22. doi:10.1073/pnas.89.1.20

[9] W. Kauzmann, "Some Factors in the Interpretation of Protein Denaturation," Advances in Protein Chemistry, Vol. 14, 1959, pp. 1-63. doi:10.1016/S0065-3233(08)60608-7

[10] A. Ben-Naim, "Strong Forces between Hydrophilic Macromolecules; Implications in Biological Systems," Journal of Chemical Physics, Vol. 93, No, 25, 1991, pp. 81968210.

[11] A. Ben-Naim, "Solvent Effects on Protein Association and Protein Folding," Biopolymers, Vol. 29, No. 3, 1990, pp. 567-596. doi:10.1002/bip.360290312 
[12] A. Ben-Naim, "Molecular Recognition-Viewed Through the Eyes of the Solvent," Biophysical Chemistry, Vol. 101-102, No. 10, 2002, pp. 309-319. doi:10.1016/S0301-4622(02)00165-5

[13] A. Ben-Naim, “On the Driving Forces for Protein-Protein
Association,” Journal of Chemical Physics, Vol. 125, No, 2, 2006, pp. 1-10.

[14] H. Wang and A. Ben-Naim, "Solvation and Solubility of Globular Proteins,” Journal of Physical Chemistry B, Vol. 101, No, 6, 1997, pp. 1077-1086. doi:10.1021/jp961591b 